\title{
Angiogenesis inhibitors in gastric cancer
}

\author{
This article was published in the following Dove Press journal: \\ Orphan Drugs: Research and Reviews \\ 14 July 2014 \\ Number of times this article has been viewed
}

\author{
Timothy McCarthy' \\ Bert H O'Neil ${ }^{2}$ \\ 'Division of Hematology/Oncology, \\ Department of Medicine, University \\ of North Carolina at Chapel Hill, \\ North Carolina, USA; ${ }^{2}$ Indiana \\ University School of Medicine, Melvin \\ and Bren Simon Cancer Center, \\ Indianapolis, Indiana, USA
}

\begin{abstract}
In the United States in 2013 it was estimated that 21,600 people would be diagnosed, and 10,990 would die of gastric cancer, the 14th most common type of cancer in the United States. Unfortunately, the majority of patients in Western countries present with metastatic disease, with very limited prognosis and relatively few treatment options. In recent years there has been increased interest in targeted agents in advanced gastric cancer. A common area of interest in anti-tumor therapy involves anti-angiogenic strategies. Abnormal neo-angiogenesis is a feature of many tumor types, and anti-angiogenic therapy has shown efficacy. The purpose of this article is to review the use of various angiogenesis inhibitors in gastric cancer. In addition, we will discuss in further detail ramucirumab, a new vascular endothelial growth factor receptor- 2 inhibitor which has recently been approved by the US Food and Drug Administration for treatment of advanced stomach cancer or gastroesophageal junction adenocarcinoma.
\end{abstract}

Keywords: gastric cancer, anti-angiogenic, ramucirumab, VEGFR2

\section{Introduction}

In 2013 it was estimated that 21,600 people would be diagnosed, and 10,990 would die of gastric cancer, the 14 th most common type of cancer in the United States. ${ }^{1,2}$ Gastric cancer prognosis is related to stage, including nodal involvement and extension through the gastric wall, with a greater than $50 \%$ cure rate in localized distal gastric cancer. ${ }^{3,4}$ Unfortunately, the majority of patients in Western countries present with metastatic disease, with early stage disease accounting for only $10 \%-20 \%$. Overall survival at 5 years post-diagnosis ranges from approximately $63 \%$ for localized gastric cancer confined to the primary site, to $28 \%$ for spread to regional lymph nodes, and $3.9 \%$ for distal metastatic disease. ${ }^{5}$

In recent years there has been increased interest in targeted agents in advanced gastric cancer. Only one targeted therapy, trastuzumab, is approved for use in gastric cancer, and is used in only the very small minority of patients who exhibit human epidermal growth factor receptor-2 (HER2) amplification by fluorescence in situ hybridization (FISH). A common area of interest in anti-tumor therapy involves antiangiogenic strategies. Abnormal neo-angiogenesis is a feature of many tumor types, and anti-angiogenic therapy has shown efficacy in multiple tumor types including colorectal cancer (CRC), non-small-cell lung carcinoma (NSCLC), glioblastoma, renal cell carcinoma, and ovarian epithelial cancers. Angiogenesis is driven at least in part by the interaction between vascular endothelial growth factors (VEGFs) and vascular endothelial growth factor receptors (VEGFRs). The VEGF family is made up of six members: VEGF-A, VEGF-B, VEGF-C, VEGF-D, VEGF-E, and placental
Correspondence: Bert H O'Neil Indiana University Simon Cancer Center, 535 Barnhill Dr., Room RTI30D, Indianapolis, IN 46202, USA

Email bhoneil@iu.edu 
growth factor (PlGF), along with three associated receptors, VEGFR1 (fms-like tyrosine kinase-1), VEGFR2 (kinase insert domain-containing receptor [KDR]), and VEGFR3. ${ }^{6,7}$ Angiogenesis is predominantly driven by VEGFR1 and VEGFR2 with VEGFR3 involved in lymphogenesis. The interaction of VEGF/VEGFR2 appears to be the main driver of tumor angiogenesis, leading to proliferation, migration and vascular endothelial cell differentiation. ${ }^{6,8}$

Increased expression of VEGF in tumor and serum has been associated with poor prognosis in gastric cancer, forming a rationale for study of VEGF/VEGFR inhibiting agents. There are a number of studies that have investigated the role of VEGF and prognosis in gastric cancer. These were summarized in a recent meta-analysis. ${ }^{9}$ It has been noted that previous studies that have investigated the relationship between VEGF overexpression with clinical outcome in patients with gastric cancer have often presented conflicting results. In the meta-analysis, survival data for 30 studies ( $n=3,999$ patients) was aggregated and quantitatively analyzed. Combined hazard ratios (HRs) suggested VEGF-A overexpression was linked to decreased overall survival (OS) ( $\mathrm{HR}=1.49,95 \%$ confidence interval $[\mathrm{CI}]: 1.22-1.77)$ and disease free survival (DFS) ( $\mathrm{HR}=1.85,95 \% \mathrm{CI}: 1.38-2.32)$ in patients with gastric cancer. In addition VEGF-D was also found to be an unfavorable indicator of OS ( $\mathrm{HR}=1.68,95 \%$ CI: $1.02-2.34)$ and DFS (HR $=1.88,95 \% \mathrm{CI}: 1.07-2.70)$, while VEGF-C overexpression did not correlate with OS (HR $=1.24,95 \% \mathrm{CI}: 0.92-1.56)$ or DFS (HR $=1.15,95 \%$ CI: $0.78-1.52){ }^{9}$

\section{Targeting of VEGF ligands Bevacizumab}

The first agent targeting the VEGF axis was bevacizumab, a monoclonal antibody which binds VEGF-A, resulting in its destruction and therefore preventing its binding to VEGFR1 and VEGFR2. It has shown extension of overall survival in metastatic CRC as well as prolongation of progression-free survival (PFS) and improvement in response rates in most other cancer types. Multiple Phase II single arm studies of bevacizumab in combination with first line chemotherapy in advanced gastric cancer showed the drug was well tolerated, and led to a median overall survival of nearly 17 months (Table 1). ${ }^{10-14}$

The first large randomized trial that attempted to validate promising Phase II results was the Phase III randomized Avastin $^{\circledR}$ (Hoffman-La Roche Ltd, Basel, Switzerland) in gastric cancer (AVAGAST) study. ${ }^{15}$ In the study, 774 patients were randomized to cisplatin/fluoropyrimidine combination chemotherapy plus or minus bevacizumab. This study showed improvement in response rate (46\% versus [vs] $37 \%$; $P=0.0315)$ and PFS (6.7 vs 5.3 months; HR $=0.80 ; 95 \% \mathrm{CI}$ : $0.68-0.93 ; P=0.0037$ ) for the bevacizumab arm; however, there was no improvement in overall survival. The median survival was 10.1 months compared to 12.2 months in the bevacizumab arm, a difference that was not statistically significant $(\mathrm{HR}=0.87, P=0.1002)$. Of note there appeared to be geographic regional variations in outcomes when stratified for Asian, European, and combined North and South American populations, whereby patients from the US and Europe appeared to derive benefit from bevacizumab, and Asian patients did not. The reason for this variation is currently unclear and perhaps related to underlying tumor biology differences, or differences in treatment patterns. One potential explanation is the preponderance of proximal and diffuse-type gastric cancers in Western patients. A secondary analysis of the AVAGAST study suggested that Western patients with diffuse or proximal disease were more likely to benefit from bevacizumab, although this analysis was retrospective in nature. $^{16}$

In addition, the AVAGAST study included a prospective biomarker program to investigate the role that angiogenic markers may play in predicting response to bevacizumab. Patients enrolled in the study had blood and tumor tissue samples collected at baseline, with plasma available from 712 (92\%) and tumor samples available from 727 (94\%). Pre-specified biomarkers included plasma VEGF-A, protein

Table I Trials of bevacizumab in gastric cancer

\begin{tabular}{|c|c|c|c|c|c|}
\hline Author & Agents & $\mathbf{n}$ & $\mathbf{R} \mathbf{R}$ & $\begin{array}{l}\text { PFS/TTP } \\
\text { (months) }\end{array}$ & $\begin{array}{l}\text { OS } \\
\text { (months) }\end{array}$ \\
\hline Shah et al" & Bevacizumab + docetaxel/cisplatin/5-FU & 44 & $67 \%$ & 12 & 16.8 \\
\hline Shah et $\mathrm{al}^{12}$ & Bevacizumab + cisplatin/irinotecan & 47 & $65 \%$ & 8.3 & 12.3 \\
\hline Enzinger et $\mathrm{al}^{13}$ & Bevacizumab + docetaxel/cisplatin/irinotecan & 32 & $63 \%$ & NS & NS \\
\hline El-Rayes et al $^{14}$ & Bevacizumab + docetaxel/oxaliplatin & 38 & $42 \%$ & 6.6 & I I.I \\
\hline Ohtsu et $\mathrm{al}^{15}$ & Bevacizumab + cisplatin/capecitabine & 387 & $46 \%$ & 6.7 & 12.2 \\
\hline Ohtsu et al $^{15}$ & Placebo + cisplatin/capecitabine & 387 & $37 \%$ & 5.3 & 10.1 \\
\hline
\end{tabular}

Abbreviations: 5-FU, fluorouracil; n, number of subjects; RR, response rate; PFS, progression-free survival; TTP, time to progression; OS, overall survival; NS, not specified. 
Table 2 Phase III and II studies of ramucirumab in gastric cancer

\begin{tabular}{|c|c|c|c|c|c|c|c|}
\hline Study & Agents & Setting & Phase & $\mathbf{n}$ & HR & $P$-value & OS (months) \\
\hline $\begin{array}{l}\text { NCT009I7384 } \\
\text { (REGARD) }\end{array}$ & $\begin{array}{l}\text { Ramucirumab } \\
\text { versus best } \\
\text { supportive } \\
\text { care }\end{array}$ & $\begin{array}{l}\text { Metastatic gastric or gastroesophageal junction } \\
\text { adenocarcinoma following disease progression } \\
\text { on first-line platinum- or fluoropyrimidine- } \\
\text { containing combination therapy }\end{array}$ & III & 355 & 0.776 & 0.047 & $\begin{array}{l}5.2 \text { (ramucirumab) } \\
\text { versus } 3.8 \text { (placebo) }\end{array}$ \\
\hline $\begin{array}{l}\text { NCTOII } 70663 \\
\text { (RAINBOW) }\end{array}$ & $\begin{array}{l}\text { Paclitaxel } \pm \\
\text { ramucirumab }\end{array}$ & $\begin{array}{l}\text { Metastatic gastric adenocarcinoma, refractory } \\
\text { to or progressive after first-line therapy } \\
\text { with platinum and fluoropyrimidine }\end{array}$ & III & 665 & 0.807 & 0.0169 & $\begin{array}{l}9.63 \text { (paclitaxel }+ \\
\text { ramucirumab) versus } \\
7.36 \text { (paclitaxel) }\end{array}$ \\
\hline NCTOI 246960 & $\begin{array}{l}\text { FOLFOX6 } \pm \\
\text { ramucirumab }\end{array}$ & $\begin{array}{l}\text { Advanced adenocarcinoma of the esophagus, } \\
\text { gastroesophageal junction or stomach }\end{array}$ & III & $\begin{array}{l}166 \\
\text { (estimated) }\end{array}$ & \multicolumn{3}{|c|}{ Study ongoing; results pending } \\
\hline NCT0I983878 & Ramucirumab & $\begin{array}{l}\text { Metastatic gastric or gastroesophageal junction } \\
\text { adenocarcinoma following disease progression on } \\
\text { first line platinum- or fluoropyrimidine-containing } \\
\text { combination therapy in Japanese patients }\end{array}$ & II & $\begin{array}{l}33 \\
\text { (estimated) }\end{array}$ & \multicolumn{3}{|c|}{ Study ongoing; results pending } \\
\hline
\end{tabular}

Abbreviations: $n$, number of subjects; HR, hazard ratio; OS, overall survival.

expression of neuropilin-1, and VEGFR1 and VEGFR2. A Cox proportional hazards model was used to assess correlations between biomarkers and clinical outcomes. Both baseline plasma VEGF-A levels and expression of tumor neuropilin-1 were identified as potential predictors of bevacizumab efficacy. A trend toward improved overall survival was noted in patients with high baseline plasma VEGF-A levels (HR, 0.72; 95\% CI, 0.57 to 0.93 ) compared to patients with low VEGF-A levels (HR, $1.01 ; 95 \% \mathrm{CI}, 0.77$ to 1.31 ; interaction $P=0.07$ ). A negative correlation was found with improved overall survival in patients with lower expression of neuropilin-1 (HR, 0.75; 95\% CI, 0.59 to 0.97 ) compared to those with high expression (HR, $1.07 ; 95 \% \mathrm{CI}, 0.81$ to 1.40 ; interaction $P=0.06$ ). Of note, these results were significant only in non-Asian region patients. ${ }^{17}$ Whether bevacizumab will be re-explored in a purely Western gastric cancer population is unclear at this time.

Aflibercept is another VEGF ligand binder, but with a potentially important difference compared with bevacizumab. Aflibercept acts as a soluble VEGF receptor decoy with affinity for VEGF-A, VEGF-B, and PlGF. Early phase studies showed efficacy and drug tolerability with common grade 3 or 4 toxicities of hypertension, proteinuria, thrombosis, fatigue, leukopenia and hoarse voice. ${ }^{6,18-21}$ Although it has demonstrated efficacy in treatment of refractory metastatic CRC, it has not shown benefit in NSCLC, prostate or pancreatic adenocarcinoma. ${ }^{22-26} \mathrm{Aflibercept}$ is currently being investigated in a Phase II clinical trial to test its safety and efficacy in combination with mFOLFOX6 compared to $\mathrm{mFOL-}$ FOX6 alone in patients with previously-untreated advanced esophagogastric adenocarcinoma. The desired primary outcome will be PFS. Secondary outcome measures will include safety and tolerability, evaluating toxicity, and number of adverse events. ${ }^{27}$

\section{VEGF receptor tyrosine kinase inhibitors}

There are several United States Food and Drug Administration (FDA)-approved small molecule tyrosine kinase inhibitors (TKIs) that target the VEGF receptors. The mechanism of action of TKIs is through targeting the binding of adenosine triphosphate (ATP) to the tyrosine domain of VEGFRs in a reversible manner. Unfortunately due to lack of specificity, most of these drugs also inhibit other receptors in addition to VEGF blockade including platelet-derived growth factor (PDGF), the protein-coding KIT gene, and often many others. It is likely that these off-target effects cause TKIs to have increased toxicity when compared to VEGF ligand inhibitors. ${ }^{22,28}$ Additionally, these agents generally produce incomplete blockade of angiogenic kinases which may affect efficacy.

Two examples of these TKIs include the drugs sunitinib and sorafenib. Both of these drugs cause the hypertension that is common to all VEGF/VEGFR targeting agents, but additionally seen are off-target toxicities such as weakness, nausea, hand-foot syndrome, rash, diarrhea, hypothyroidism, and transaminitis. ${ }^{6,29,30}$ Sunitinib has been studied in a Phase II, open-label, multi-center study in patients with advanced gastric or gastroesophageal junction adenocarcinoma who had previously been treated with chemotherapy. The primary end point was objective response rate, with secondary end points including clinical benefit rate, duration of response, PFS, OS, pharmacokinetics, pharmacodynamics, safety and tolerability, and quality of life. Seventy-eight patients were enrolled, with $93.6 \%$ having gastric adenocarcinoma and $93.6 \%$ with metastatic disease. Two patients (2.6\%) showed partial response, and 25 patients (32.1\%) had a best response of stable disease $\geq 6$ weeks. The median PFS was 2.3 months (95\% CI, 1.6-2.6 months) and median OS 
was 6.8 months (95\% CI, 4.4-9.6 months). Adverse events reported included grade 3 or greater thrombocytopenia in $34.6 \%$ and neutropenia in $29.4 \%$ of patients. The other most common adverse events included fatigue, anorexia, nausea, diarrhea and stomatitis. The study authors concluded that although sunitinib had insufficient clinical value as a single agent therapy in the second line setting, given its progression, delaying effect, and favorable toxicity profile it warrants further study in combination with chemotherapy. ${ }^{31}$

Sorafenib has been investigated in combination with cisplatin and capecitabine in a Phase I dose escalation study in patients with advanced gastric cancer. This trial showed a response rate of $63 \%$, PFS of 10 months, and OS of 15 months. A Phase II study of sorafenib combined with cisplatin and docetaxel used in the first line setting showed a response rate of $41 \%$, and an OS of 13.6 months. Adverse events included grade 3 or greater neutropenia in $64 \%$ of patients. ${ }^{10}$ There are currently no ongoing Phase III trials of either of these agents.

\section{Ramucirumab}

Given the above limitations, the drug ramucirumab has been investigated as an alternative method of anti-VEGF receptor therapy with higher specificity for VEGFR2 blockade than the previously mentioned TKIs. Ramucirumab is a fully human immunoglobulin G-subclass 1 (IgG1) monoclonal antibody that is a specific and potent inhibitor of VEGFR2. It binds to the VEGF-binding domain of VEGFR2, inhibiting the VEGF-VEGFR2 complex, and has been shown to decrease tumor vascularity in preclinical data. ${ }^{32}$ This mechanism is in contradistinction to that of bevacizumab, which binds to the VEGF ligand itself, destroying the ligand. Theoretically, ramucirumab may have an advantage when compared to bevacizumab, which binds to VEGF-A only. Proteolytic processing of VEGF-C and VEGF-D allows them to bind to VEGFR2 and promote angiogenesis, which would allow for a bypass of bevacizumab's mechanism of action, whereas ramucirumab would work at the level of the VEGFR2 receptor itself, preventing all known VEGFs from binding. ${ }^{22}$

A number of Phase I and II studies of ramucirumab have been completed in various solid tumor types including gastric carcinoma, breast cancer, non-small cell lung carcinoma, hepatocellular carcinoma, metastatic melanoma, colorectal cancer, ovarian cancer and prostate cancer. ${ }^{6} \mathrm{~A}$ Phase I clinical trial reported by Spratlin et $\mathrm{al}^{32}$ reported on pharmacokinetics, pharmacodynamics and efficacy in patients with advanced solid malignancies. Patients were treated with once weekly escalating doses of ramucirumab in a $3+3$ dose escalation trial. In the study, 37 patients were treated over a dosage range of 2 to $16 \mathrm{mg} / \mathrm{kg}$. A trough level of $20 \mu \mathrm{g} / \mathrm{mL}$ was targeted. ${ }^{32}$ Half-life ranged between 200 and 300 hours. The drug showed a nonlinear relationship between dosing and clearance rate. There was disproportionate decrease in drug clearance with increasing dose, thought to be due to saturation of VEGFR2 as the primary clearance mechanism. ${ }^{6,32}$ The maximum tolerated dose was determined to be $13 \mathrm{mg} / \mathrm{kg}$ after two patients developed dose-limiting hypertension and venous thrombosis at the $16 \mathrm{mg} / \mathrm{kg}$ dose. Other major toxicities included grade 3 fatigue, nausea, vomiting, and proteinuria. A partial response (PR) was seen in four of 27 patients with measurable disease including gastric cancer, melanoma, ovarian, and uterine cancer. Overall a PR or stable disease was seen in approximately $73 \%$ of patients with eleven of 37 patients showing either PR or stable disease at 6 months out, suggestive of substantial single-agent activity. ${ }^{32}$

Ramucirumab has been tested in two Phase III clinical trials focusing on advanced gastric carcinoma in the second line setting. These are the REGARD and RAINBOW (US National Institutes of Health clinical trial NCT01170663) trials (Table 2). The REGARD study was an international, randomized, double-blind, placebo-controlled Phase III trial in patients with advanced gastric or gastroesophageal junction adenocarcinoma and disease progression after first-line platinum- or fluoropyrimidine-containing chemotherapy. The primary study end point was overall survival, with intention to treat analysis. Patients were randomly assigned (2:1) to best supportive care plus either ramucirumab at $8 \mathrm{mg} / \mathrm{kg}$ or placebo, given intravenously every 2 weeks. A total of 355 patients were randomized, with 238 receiving ramucirumab and 117 receiving placebo. The median overall survival was found to be 5.2 months in the ramucirumab arm compared to 3.8 months in the placebo arm (HR 0.776, 95\% CI 0.603-0.998; $P=0.047)$ and disease control rate was improved in the ramucirumab arm (49\% vs $23 \%)$. No radiographic responses were seen in either arm. Toxicities seen included fatigue (36\% in the ramucirumab arm compared to $40 \%$ in the placebo arm), hypertension ( $16 \%$ vs $8 \%$ ), and rates of other adverse events were mostly similar between groups (223 [94\%] vs 101 [88\%]) between the ramucirumab and placebo arms. ${ }^{34}$

It is very interesting that REGARD showed positive results while AVAGAST ${ }^{15}$ did not in terms of OS. On subset analysis of AVAGAST, survival benefit for bevacizumab was limited to non-Asian patients. In REGARD this was not the case, with the survival benefit from ramucirumab similar between Asian patients and those from America, Europe, and Australia. 
That said, there were relatively few Asian patients enrolled in REGARD ( $16 \%$ in both ramucirumab and placebo arms), so this difference alone could potentially explain the difference between the two trials. ${ }^{11}$ Another potential contributing factor may be that the mechanism of action of ramucirumab is significantly different enough to surpass the activity of bevacizumab. This may be further supported by the fact that ramucirumab demonstrates single-agent activity, whereas bevacizumab and aflibercept have failed to do so. Lastly, it is possible that bevacizumab would have similar activity if tested alone in a second-line setting.

In comparison to the REGARD trial, the Phase III COUGAR-02 study (NCT00978549) ${ }^{35}$ evaluated docetaxel plus active symptom control (ASC) in relapsed esophagogastric cancer. The results of COUGAR-02 proved to be very similar to those of ramucirumab, with COUGAR-02 showing that the addition of docetaxel to ASC provided a median overall survival benefit of 1.6 months (5.2 months versus 3.6 months with ASC alone; HR $=0.67, P=0.01) .{ }^{35}$ Based on the results of the COUGAR-02 study, which highlight the benefit that patients get from an active second-line therapy, there remains the question of whether ramucirumab will demonstrate an additive benefit when combined with an active agent in gastric cancer. The RAINBOW trial seeks to answer this question.

RAINBOW was a Phase III study comparing the safety and efficacy of paclitaxel plus ramucirumab versus paclitaxel plus placebo with a primary end point of overall survival and secondary end points of progression-free survival, time to progressive disease, objective response, and safety in the advanced gastric cancer population. Data for the RAINBOW trial were presented as an abstract at the 2014 American Society of Clinical Oncology (ASCO) Gastrointestinal Cancers Symposium. The study included 665 patients with disease progression on first-line platinum/fluoropyrimidine-based combination therapy. The addition of ramucirumab to paclitaxel significantly prolonged the primary end point of overall survival from a median of 7.36 to 9.63 months $(P=0.0169)$. The difference between arms translated into a $19 \%$ reduction in the risk of death with inclusion of ramucirumab ( $\mathrm{HR}=0.807,95 \% \mathrm{CI}$ : $0.678-0.962$ ). PFS was also prolonged from 2.86 months to 4.40 months $(\mathrm{HR}=0.635,95 \%$ CI: $0.536-0.752 ; P<0.0001)$. Inclusion of ramucirumab also increased the overall response rate compared with paclitaxel alone $(28 \%$ versus $16 \% ; P=0.0001)$ and significantly increased the disease control rate $(80 \%$ versus $64 \% ; P<0.0001) .{ }^{36}$

Ramucirumab in combination with paclitaxel was relatively well tolerated. Compared to paclitaxel alone there was a greater incidence of grade 3 or greater neutropenia (40.7\% versus $18.8 \%$ with paclitaxel alone), leukopenia (17.4\% versus $6.7 \%$ with paclitaxel alone), hypertension ( $14.7 \%$ versus $2.7 \%$ with paclitaxel alone), and fatigue (11.9\% versus 5.5\% with paclitaxel alone). Of note these adverse events did not lead to increased treatment discontinuation in the ramucirumab arm. The rates of treatment-related deaths were also not significantly different between the two arms ( $4.0 \%$ with ramucirumab/paclitaxel versus $4.6 \%$ with paclitaxel alone). The incidence of febrile neutropenia was also similar between the two arms (3.1\% with ramucirumab/ paclitaxel versus $2.4 \%$ with paclitaxel alone). ${ }^{36}$

Ramucirumab is currently being investigated for first line use in combination with chemotherapy in gastric cancer. A Phase II study (NCT01246960) is focused on previouslyuntreated advanced esophageal, gastric and gastroesophageal junction carcinoma. It is a randomized, placebo-controlled, double-blind Phase II study of mFOLFOX6 chemotherapy plus ramucirumab versus mFOLFOX6, with the primary outcome of progression-free survival. ${ }^{37}$

Based on the results of the REGARD trial, the FDA approved ramucirumab on April 21, 2013 to treat patients with advanced stomach cancer or gastroesophageal junction adenocarcinoma. The FDA reviewed ramucirumab under its priority review program, which allows an expedited review for drugs with the potential to be a significant improvement in safety or effectiveness in the treatment of a serious condition. Ramucirumab was also granted orphan product designation because it is intended to treat a rare disease or condition. ${ }^{38}$

\section{Conclusion}

The field of anti-angiogenesis drugs continues to be an area of interest in cancer in general and in gastric cancer specifically. The success of ramucirumab in this disease should open the door to continued study of novel antiangiogenic strategies. In addition to new drug development, a search for predictive biomarkers that would identify gastric cancer patients who might benefit from a given treatment will continue to be necessary. Through continued study and drug development, coupled with improving understanding of individual patient characteristics, it is hoped that angiogenesis in gastric cancer will continue to be an effective therapeutic target, and ultimately translate into further improvements in survival.

\section{Disclosure}

The authors report no conflict of interest in this work.

\section{References}

1. American Cancer Society. Cancer Facts and Figures 2013. Atlanta, GA, USA: American Cancer Society; 2013. Available from: http://www. cancer.org/acs/groups/content/@epidemiologysurveillance/documents/ document/acspc-036845.pdf. Accessed June 8, 2014. 
2. Kurtz RC, Sherlock P. The diagnosis of gastric cancer. Semin Oncol. 1985;12(1):11-18.

3. Siewert JR, Bottcher K, Stein HJ, et al. Relevant prognostic factors in gastric cancer: ten-year results of the German Gastric Cancer Study. Ann Surg. 1998;228(4):449-461.

4. Nakamura K, Ueyama T, Yao T, et al. Pathology and prognosis of gastric carcinoma. Findings in 10,000 patients who underwent primary gastrectomy. Cancer. 1992;70(5):1030-1037.

5. Howlader N, Noone AM, Krapcho, et al. SEER Cancer Statistics Review, 1975-2010. National Cancer Institute, Bethesda MD, USA. Available from: http://seer.cancer.gov/csr/1975_2010/. Based on Nov 2012 SEER data posted to the SEER web site 2013. Accessed October 1, 2013.

6. Spratlin J. Ramucirumab (IMC-1121B): Monoclonal antibody inhibition of vascular endothelial growth factor receptor-2. Curr Oncol Rep. 2011;13(2):97-102.

7. Veikkola T, Alitalo K. VEGFs, receptors and angiogenesis. Semin Cancer Biol. 1999;9(3):211-220.

8. Gille H, Kowalski J, Li B, et al. Analysis of biological effects and signaling properties of Flt-1 (VEGFR-1) and KDR (VEGFR-2). A reassessment using novel receptor-specific vascular endothelial growth factor mutants. J Biol Chem. 2001;276(5):3222-3230.

9. Peng L, Fang W, Zhao P. Prognostic significance of vascular endothelial growth factor immunohistochemical expression in gastric cancer: a meta-analysis. Mol Biol Rep. 2012;39:9473-9484.

10. Smyth EC, Cunningham D. Targeted therapy for gastric cancer. Curr Treat Options Oncol. 2012;3:377-389.

11. Shah MA, Jhawer M, Ilson DH, et al. Phase II study of modified docetaxel, cisplatin and fluorouracil with bevacizumab in patients with metastatic gastroesophageal adenocarcinoma. J Clin Oncol. 2011;29(7):868-874.

12. Shah MA, Ramanathan RK, Ilson DH, et al. Multicenter Phase II study of irinotecan, cisplatin, and bevacizumab in patients with metastatic gastric or gastroesophageal junction adenocarcinoma. J Clin Oncol. 2006;24(33):5201-5206.

13. Enzinger PC, Ryan DP, Regan EM. Phase II trial of docetaxel, cisplatin, irinotecan and bevacizumab in metastatic esophagogastric cancer [abstract]. J Clin Oncol. 2008;26(15):4552.

14. El-Rayes BF, Zalupski M, Bekai-Saab T, et al. A Phase II study of bevacizumab, oxaliplatin, and doectaxel in locally advanced and metastatic gastric and gastroesophageal junction cancers. Ann Oncol. 2010;21(10):1999-2004.

15. Ohtsu A, Shah MA, Van Cutsem E, et al. Bevacizumab in combination with chemotherapy as first-line therapy in advanced gastric cancer: a randomized, double-blind, placebo-controlled Phase III study. J Clin Oncol. 2011;29(30):3968-3976.

16. Shah MA, Van Cutsem E, Kang YK, et al. Survival analysis according to disease subtype in AVAGAST: first-line capecitabine and cisplatin plus bevacizumab or placebo in patients with advanced gastric cancer [abstract]. J Clin Oncol. 2012;30(4):5.

17. Van Cutsem E, de Haas S, Kang Y. Bevacizumab in combination with chemotherapy as first-line therapy in advanced gastric cancer: a biomarker evaluation from the AVAGAST randomized Phase III trial. J Clin Oncol. 2012;30(17):2119-2127.

18. Holash J, Davis S, Papadopoulos N, et al. VEGF-Trap: a VEGF blocker with potent antitumor effects. Proc Natl Acad Sci U SA. 2002;99(17): 11393-11398.

19. Byrne AT, Ross L, Holash J, et al. Vascular endothelial growth factortrap decreases tumor burden, inhibits ascites, and causes dramatic vascular remodeling in an ovarian cancer model. Clin Cancer Res. 2003;9(15):5721-5728.

20. Tew WP, Gordon M, Murren J, et al. Phase I study of aflibercept administered subcutaneously to patients with advanced solid tumors. Clin Cancer Res. 2010;16(1):358-366.

21. Lockart AC, Rothenberg ML, Dupont J, et al. Phase I study of intravenous vascular endothelial growth factor trap, aflibercept, in patients with advanced solid tumors. J Clin Oncol. 2010;28(2):207-214.
22. Clarke JM, Hurwitz H. Targeted inhibition of VEGF receptor 2: an update on ramucirumab. Expert Opin Biol Ther. 2013;13(8):1187-1196.

23. Van Cutsem E, Tabernero J, Lakomy R, et al. Addition of aflibercept to fluorouracil, leucovorin, and irinotecan improves survival in a Phase III randomized trial in patients with metastatic colorectal cancer previously treated with an oxaliplatin-based regimen. J Clin Oncol. 2012;30(28):3499-3506.

24. Ramlau R, Gorbunova V, Ciuleanu TE, et al. Aflibercept and docetaxel versus docetaxel alone after platinum failure in patients with advanced or metastatic non-small-cell lung cancer: a randomized, controlled Phase III trial. J Clin Oncol. 2012;30 (29):3640-3647.

25. Regeneron. Sanofi and Regeneron announce regulatory and clinical update for ZALTRAP ${ }^{\circledR}$ (aflibercept) [press release]. April 5, 2012. Available from: http://investor.regeneron.com/releasedetail. cfm?releaseid=661995. Accessed June 9, 2014.

26. Regeneron Pharmaceuticals, Inc. Phase III trial of aflibercept in metastatic pancreatic cancer discontinued September 11, 2009 [press release]. September 11, 2009. Available from: http://newsroom. regeneron.com/releasedetail.cfm?ReleaseID $=408744$. Accessed June 9, 2014.

27. Enzinger P, Dana-Farber Cancer Institute. Randomized, double-blind, placebo controlled Phase II study of FOLFOX \pm Ziv-Aflibercept in patients with advanced esophageal and gastric cancer. Available from: http://clinicaltrials.gov/ct2/show/study/NCT01747551. NLM Identifier: NCT01747551. Accessed June 9, 2014.

28. Ivy SP, Wick JY, Kaufman BM. An overview of small-molecule inhibitors of VEGFR signaling. Nat Rev Clin Oncol. 2009; 6(10):569-579.

29. Awada A, Hendlisz A, Gil T, et al. Phase I safety and pharmacokinetics of BAY 43-9006 administered for 21 days on/7 days off in patients with advanced, refractory solid tumours. Br J Cancer. 2005;92(10): 1855-1861.

30. Faivre S, Delbaldo C, Vera K, et al. Safety, pharmacokinetic, and antitumor activity of SU11248, a novel oral multitarget tyrosine kinase inhibitor, in patients with cancer. J Clin Oncol. 2006;24(1):25-35.

31. Bang YJ, Kang YK, Kang WK, et al. Phase II study of sunitinib as second-line treatment for advanced gastric cancer. Invest New Drugs. 2011;29:1449-1458.

32. Spratlin JL, Cohen RB, Eadens M, et al. Phase I pharmacologic and biologic study of ramucirumab (IMC-1121B), a fully human immunoglobulin G1 monoclonal antibody targeting the vascular endothelial growth factor receptor-2. J Clin Oncol. 2010;28(5):780-787.

33. Wadhwa R, Taketa T, Sudo K, et al. Ramucirumab: a novel antiangiogenic agent. Future Oncol. 2013;9(6):789-795.

34. Fuchs C, Tomasek J, Yong CJ, et al. Ramucirumab monotherapy for previously treated advanced gastric or gastro-oesophageal junction adenocarcinoma (REGARD): an international, randomised, multicentre, placebo-controlled, Phase 3 trial. Lancet. 2014;383(9911):31-39.

35. Ford H, Marshall A, Wadsley J, et al. Cougar-02: A randomized Phase III study of docetaxel versus active symptom control in advanced esophagogastric adenocarcinoma [abstract]. J Clin Oncol. 2013; 31(Suppl 4; abstr LBA4).

36. Wilke H, Van Cutsem E, Oh SC, et al. RAINBOW: A global, Phase III, randomized, double-blind study of ramucirumab plus paclitaxel versus placebo plus paclitaxel in the treatment of metastatic gastroesophageal junction (GEJ) and gastric adenocarcinoma following disease progression on first-line platinum- and fluoropyrimidine-containing combination therapy rainbow IMCL CP12-0922 (I4T-IE-JVBE). J Clin Oncol. 2014;32(Suppl 3; abstr LBA7).

37. Eli Lilly. A study of ramucirumab in patients with gastric, esophageal and gastroesophageal cancer. Available from: http://clinicaltrials.gov/ ct2/show/record/NCT01246960. Accessed June 10, 2014

38. US Food and Drug Administration. FDA approves Cyramza for stomach cancer. [press release]. Silver Spring, MD: US Food and Drug Administration; April 21, 2014. Available from: http://www.fda. gov/NewsEvents/Newsroom/PressAnnouncements/ucm394107.htm. Accessed June 12, 2014. 


\section{Publish your work in this journal}

Orphan Drugs: Research and Reviews is an international, peer-reviewed, open access journal publishing original research, reports, reviews and commentaries on all areas of the design and development of orphan drugs for the treatment of rare diseases through to clinical applications Clinical outcomes, patient safety, and programs for the development and

Submit your manuscript here: http://www.dovepress.com/orphan-drugs-research-and-reviews-journ effective, safe, and sustained use of medicines will be a feature of the journal. The manuscript management system is completely online and includes a very quick and fair peer-review system, which is all easy to use. Visit http://www.dovepress.com/testimonials.php to read real quotes from published authors. 